\title{
How shall we treat locally advanced triple negative breast
}

\section{cancer? [version 1; peer review: 1 approved]}

\section{Paulo Luz (D), David Dias, Ana Fortuna, Luis Bretes, Beatriz Gosalbez}

Medical Oncology, Centro Hospitalar Universitário do Algarve, Faro, Portugal

V1 First published: $16 \operatorname{Sep} 2019, \mathbf{8 : 1 6 4 9}$

https://doi.org/10.12688/f1000research.20509.1

Latest published: 04 May 2020, 8:1649

https://doi.org/10.12688/f1000research.20509.2

\section{Abstract}

Triple negative breast cancer (TNBC) has been shown to respond to neoadjuvant chemotherapy (NACT). It has been established that achieving pathological complete response ( $p C R$ ) for certain aggressive subtypes of breast cancer, including HER-2 (over-expressed) and TNBC, provides an important surrogate marker for predicting longterm clinical response and survival outcomes.

How to increase the number of patients that achieve $\mathrm{pCR}$ remains challenging. Platinum-based NACT seems to be part of the solution and capecitabine, an active drug in metastatic breast cancer, but not a standard one in earlier stages may have found its place in the adjuvant setting.

\section{Keywords}

triple negative, breast cancer, neoadjuvant chemotherapy

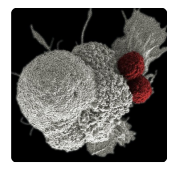

This article is included in the Oncology

gateway.

\section{Open Peer Review \\ Approval Status \\ 1 \\ 2 \\ version 2 \\ (revision) \\ 04 May 2020 \\ view \\ version 1 \\ 16 Sep 2019 \\ 1. Brian D. Lehmann, Vanderbilt University \\ Medical Center, Nashville, USA \\ 2. Bruna Cerbelli, Vanderbilt University Medical \\ Center, Nashville, USA}

Any reports and responses or comments on the article can be found at the end of the article.

Corresponding author: Paulo Luz (p_luz@msn.com)

Author roles: Luz P: Investigation, Methodology, Writing - Original Draft Preparation, Writing - Review \& Editing; Dias D: Writing Original Draft Preparation; Fortuna A: Resources; Bretes L: Supervision, Validation; Gosalbez B: Supervision, Writing - Original Draft Preparation, Writing - Review \& Editing

Competing interests: No competing interests were disclosed.

Grant information: The author(s) declared that no grants were involved in supporting this work.

Copyright: ( 2019 Luz P et al. This is an open access article distributed under the terms of the Creative Commons Attribution License, which permits unrestricted use, distribution, and reproduction in any medium, provided the original work is properly cited.

How to cite this article: Luz P, Dias D, Fortuna A et al. How shall we treat locally advanced triple negative breast cancer? [version 1; peer review: 1 approved] F1000Research 2019, 8:1649 https://doi.org/10.12688/f1000research.20509.1

First published: 16 Sep 2019, 8:1649 https://doi.org/10.12688/f1000research.20509.1 
Triple negative breast cancer (TNBC) is immunohistochemically defined as the lack of expression of estrogen, progesterone receptor and human epidermal growth factor receptor 2 (HER-2). It accounts for $15-20 \%$ of breast cancer cases and is characterized as a molecular heterogeneous disease that usually presents an aggressive clinical behavior and higher prevalence in younger women ${ }^{1}$. Once TNBC has metastasized, it has the worst prognosis and the shortest OS of all breast cancer subtypes. On the other hand, TNBCs are highly chemo-sensitive and have been shown to respond very well to neoadjuvant chemotherapy (NACT) ${ }^{1-3}$.

The main goal of NACT strategies are not only to decrease the need for radical mastectomy but also to obtain an important predictive marker of favourable prognosis - the pathological complete response (pCR) - which is defined as the absence of invasive tumour cells (ypT0/is, ypN0/is). A correlation was observed between the pCR and the overall survival (OS) and disease free survival (DFS) outcomes in all subtypes of breast cancer, specially in aggressive ones such as HER2 positive and TNBC $^{2}$. The pCR achieved by NACT represents to date the ideal endpoint in translational investigation of biomarkers and pharmacological treatments. Patients who do not have pCR after NACT with the combination of taxane and anthracycline have 20 to $30 \%$ risk of relapse $e^{2,3}$.

How to increase the number of patients that achieve pCR remains challenging. Platinum-based NACT seems to be part of the solution. Recently, a metanalysis that enrolled nine randomized clinical trials (RCT) with 2109 patients showed that platinum-based NACT compared to platinum-free NACT significantly increased pCR rate from $37.0 \%$ to $52.1 \%{ }^{4}$. However, only two RCTs (CALGB 40603 and Gepar-Sixto trial) reported survival outcomes: no significant difference in event free survival (EFS) and OS was observed ${ }^{5,6}$. In the CALGB study adding carboplatin did not significantly impact survival. The absolute benefit in 3-year event-free survival of adding carboplatin was $4.9 \%$ and the OS differences were also not significant, with $81,9 \%$ OS in the carboplatin group versus $84,6 \%$ without carboplatin. These results conflict with those in the Gepar-Sixto trial. In the TNBC subgroup, carboplatin resulted in a significantly improved pCR rate over control (53\% vs $37 \%$; $\mathrm{P}=0.005)$. This translated into an absolute benefit in 3-year event EFS for the addition of carboplatin over control of $9.7 \%(85.8 \%$ vs $76.1 \%$, respectively). What reasons can explain this discrepancy? There are several differences between these two studies worth noting. GeparSixto had more-favorable baseline characteristics, as $26 \%$ of patients were cN0, compared with $42 \%$ in CALGB 40603. Additionally, a larger proportion were cT1 in GeparSixto $(26 \%$ vs $11 \%$ ). In CALGB 40603, the backbone therapy also included cyclophosphamide, which can also cause DNA damage like platinum agents, potentially making the treatment effect similar in the control and experimental arms $\mathrm{s}^{5,6}$. Notably, BRCA-mutated patients experienced overall higher $\mathrm{pCR}$ rates, although no benefit was observed with the ${ }^{4}$.

Current controversy in this field also includes the benefit of additional therapy after surgery. Given the still significantly high rates of residual disease after neoadjuvant therapy in TNBC, which portends inferior DFS, another approach to improving outcomes in this population is to add additional adjuvant therapy after surgery. The great majority of the studies focus on capecitabine, an active drug in metastatic TNBC. In the CREATE $X$ trial (Capecitabine for Residual Cancer as Adjuvant Therapy $)^{7}$ patients who have not achieved pathologic complete response after undergoing neoadjuvant therapy were randomized to receive standard treatment either with capecitabine or without (control). Among patients with triple negative disease, the rate of disease-free survival was $69.8 \%$ in the capecitabine group versus $56.1 \%$ in the control group, and the overall survival rate was $78.8 \%$ versus $70.3 \%$. Recently, at the San Antonio Breast Cancer Symposium 2018, the results of the phase III trial conducted by the Spanish group and the Central and South American group were presented, where treating patients with early-stage triple-negative breast cancer with capecitabine after surgery and standard chemotherapy did not significantly improve disease-free or overall survival compared with observation group ${ }^{8}$. One possible explanation for the discrepancy between the results of the CREATE-X trial and this trial may be the different prognostic features between the populations. The risk of relapse of the population was much lower than in the CREATE-X trial. So, capecitabine, an active drug in metastatic breast cancer, but not a standard one in earlier stages may have found its place.

An important question remains, should standard of care with NACT on TNBC rely on platinum-based treatments with the intent of achieving higher pCR rates and a probable benefit in OS or should it continue with taxane and antracyclin based combinations and consider the use capecitabine when pCR is not feasible?

According to the authors' perspectives, the impact of platinum on pCR and OS cannot simply be ignored. This option must be considered after balancing the potential benefits on disease outcomes versus increased toxicity. Special attention must be placed in older and frail patients that are still capable and willing to undergo NACT. In this subgroup of patients, taxane and antracyclin combination remains a valid first choice treatment and adjuvant capecitabine should be considered when residual tumor is still present. In young high risk patients who underwent platinum-based NACT and didn't achieve pCR, adjuvant capecitabine should be discussed even we don't have any RCT to support this idea.

Chemotherapy remains the backbone of TNBC, but research and development of new modalities of treatment continues. Recent and promising results were available in the metastatic setting. We hope that some of these drugs, like immunotherapy, may soon have a role in the neoadjuvant setting. Right now, we know neoadjuvant treatment allows us to re-write the story right at the start and we just cannot miss that opportunity.

\section{Data availability}

Underlying data

No data are associated with this study. 
1. Harbeck N, Gnant M: Breast cancer. Lancet. 2017; 389(10074): 1134-1150. PubMed Abstract | Publisher Full Text

2. Cortazar $\mathrm{P}$, Zhang $\mathrm{L}$, Untch $\mathrm{M}$, et al:: Pathological complete response and longterm clinical benefit in breast cancer: the CTNeoBC pooled analysis. Lancet. 2014; 384(9938): 164-172.

PubMed Abstract | Publisher Full Text

3. von Minckwitz G, Untch M, Blohmer JU, et al.: Definition and impact of pathologic complete response on prognosis after neoadjuvant chemotherapy in various intrinsic breast cancer subtypes. J Clin Oncol. 2012; 30(15): 1796-1804. PubMed Abstract | Publisher Full Text

4. Poggio F, Bruzzone M, Ceppi M, et al.: Platinum-based neoadjuvant chemotherapy in triple-negative breast cancer: a systematic review and metaanalysis. Ann Oncol. 2018; 29(7): 1497-1508.

PubMed Abstract | Publisher Full Text

5. Untch $\mathrm{M}$, Schneeweiss $\mathrm{A}$, Salat $\mathrm{C}$, et al:: 163PDLong-term survival analysis of the randomized phase II trial investigating the addition of carboplatin to neoadjuvant therapy for triple-negative (TNBC) and HER2-positive early breast cancer (GeparSixto). Ann Oncol. 2017; 28(suppl_ 5).

Publisher Full Text

6. Sikov W, Berry D, Perou C, et al.: Abstract S2-05: Event-free and overall survival following neoadjuvant weekly paclitaxel and dose-dense $A C+/$ - carboplatin and/or bevacizumab in triple-negative breast cancer: Outcomes from CALGB 40603 (Alliance). Cancer Res. 2016; 76(4 Supplement): S2-05-S2-05. Publisher Full Text

7. Masuda N, Lee SJ, Ohtani S, et al:: Adjuvant Capecitabine for Breast Cancer after Preoperative Chemotherapy. N Engl J Med. 2017; 376(22): 2147-2159. PubMed Abstract | Publisher Full Text

8. Martín M, Barrios $\mathrm{CH}$, Torrecillas L, et al:: Abstract GS2-04: Efficacy results from CIBOMA/2004-01_GEICAM/2003-11 study: A randomized phase III tria assessing adjuvant capecitabine after standard chemotherapy for patients with early triple negative breast cancer. Cancer Res. 2019; 79(4 Supplement) GS2-04 LP-GS2-04.

Publisher Full Text 


\section{Open Peer Review}

\section{Current Peer Review Status:}

\section{Version 1}

Reviewer Report 21 January 2020

https://doi.org/10.5256/f1000research.22542.r58166

(C) 2020 Lehmann B. This is an open access peer review report distributed under the terms of the Creative Commons Attribution License, which permits unrestricted use, distribution, and reproduction in any medium, provided the original work is properly cited.

\section{Brian D. Lehmann}

Department of Medicine, Vanderbilt University Medical Center, Nashville, TN, USA

In this article the authors provide perspective on the use of platinum compounds in the treatment of early stage triple-negative breast cancer. The authors provide opinions on a recently published meta-analysis of nine randomized clinical trials (RCT) treated with platinum-based neoadjuvant chemotherapy.

Specific comments:

1. Please finish the sentence, "Notably, BRCA-mutated patients experienced overall higher pCR rates, although no benefit was observed with the." The sentence and idea trying to be presented is incomplete.

2. The authors state, "What reasons can explain this discrepancy? There are several differences between these two-studies worth noting. GeparSixto had more-favorable baseline characteristics, as $26 \%$ of patients were cN0, compared with $42 \%$ in CALGB 40603 ." This statement does not appear to be accurate. How is $26 \%$ of patients with cN0 in GeparSixto more favorable than $42 \%$ of patients with $\mathrm{CNO}$ in CALGB? Please revise and double check the numbers. $42 \%$ is correct for CALGB as the patient population was limited to TNBC. GeparSixto included both TNBC and HER2 positive and at least in the combined population cNO was $50 \%$. Not sure what the value is when restricted to TNBC. The authors should consider comparing tumor grade, as higher grades are associated with better chemotherapy response. In CALGB $76 \%$ of patients had tumors of high grade, while $65 \%$ of tumors were high grade in GeparSixto, albeit in both TNBC and HER2.

3. The statement, "Recently, at the San Antonio Breast Cancer Symposium 2018...)" is not so recent anymore. Consider removing "recent" or replacing with newer published data.

4. The authors propose the very relevant question, "An important question remains, should standard of care with NACT on TNBC rely on platinum-based treatments with the intent of achieving higher $\mathrm{PCR}$ rates and a probable benefit in OS or should it continue with taxane and antracyclin based combinations and consider the use capecitabine when PCR is not 
feasible?" While the authors mention the data cannot be ignored, the authors do not mention how the question might finally be resolved. Especially given that there were no EFS or OS benefits from the two studies with published results, how can we justify the substantial toxicities of an additional platinum compound? Perhaps only when we see additional long-term analysis from the majority of RCTs with platinum compounds could the question be answered.

5. The authors conclude with, "We hope that some of these drugs, like immunotherapy, may soon have a role in the neoadjuvant setting." While this may certainly be true in the metastatic setting, recent results suggest that this may not be the case in the neoadjuvant setting. Please include a discussion of immune checkpoint inhibitors in the neoadjuvant setting. Results from the NeoTRIPaPDL1 trial presented at SABC 2020 (Abstract \#GS3-04) concluded that Atezolizumab with neoadjuvant chemotherapy does not improve pathologic complete response rates for patients with triple-negative breast cancer.

Is the topic of the opinion article discussed accurately in the context of the current literature?

Yes

Are all factual statements correct and adequately supported by citations? Yes

Are arguments sufficiently supported by evidence from the published literature? Partly

Are the conclusions drawn balanced and justified on the basis of the presented arguments? Yes

Competing Interests: No competing interests were disclosed.

Reviewer Expertise: Triple-negative breast cancer, translational research and genomics

I confirm that I have read this submission and believe that I have an appropriate level of expertise to confirm that it is of an acceptable scientific standard.

Author Response 28 Apr 2020

\section{Paulo Luz}

We appreciate the time and effort that you have dedicated to providing your valuable feedback on our manuscript. New evidence about immunotherapy in early TNBC is available (NeoTRIP and KEYNOTE 522). We incorporated that information in this new version of the article.

We have also been able to incorporate changes to reflect most of the suggestions provided by you.

Competing Interests: No competing interests were disclosed. 
The benefits of publishing with F1000Research:

- Your article is published within days, with no editorial bias

- You can publish traditional articles, null/negative results, case reports, data notes and more

- The peer review process is transparent and collaborative

- Your article is indexed in PubMed after passing peer review

- Dedicated customer support at every stage

For pre-submission enquiries, contact research@f1000.com 